\title{
A Web Navigation Tool for the Blind
}

\author{
Mary Zajicek \\ Oxford Brookes University \\ Headington Campus, Gipsy Lane \\ Oxford, OX3 OBP, UK \\ +441865483683 \\ mzajicek@brookes.ac.uk
}

\author{
Chris Powell \\ Oxford Brookes University \\ Headington Campus, Gipsy Lane \\ Oxford OX3 OBP, UK \\ +441865483709 \\ cpowell@brookes.ac.uk
}

\author{
Chris Reeves \\ Royal National Institute for the \\ Blind \\ 224, Great Portland Street, \\ London, WIN 6AA, UK \\ +441713881266 \\ creeves@rnib.org.uk
}

\section{ABSTRACT}

The aim of our work is to make the wealth of information on the World Wide Web more readily available to blind people. They must be able to search efficiently for relevant information and make quick and effective decisions about the usefulness of pages they retrieve. We have built a prototype application called BrookesTalk which we believe addresses this need more fully than other Web browsers. Information retrieval techniques are used to provide a set of complementary options which summarise a Web page and enable rapid decisions about its usefulness.

\subsection{Keywords}

World wide Web, browser, blind, information retrieval, HTML

\section{INTRODUCTION}

At the Speech Project at Oxford Brookes University we have built a Web navigation tool called BrookesTalk that employs information retrieval techniques to summarise the Web page for quick orientation [4]. We discuss the evaluative feedback received from blind users.

\section{THE AIMS OF THE NAVIGATION TOOL}

Existing screen readers such as JAWS and browsers such as pwWebSpeak(ä), enable the user to move around the screen, jump to interaction objects in the screen and read particular areas of text. These tools become unwieldy when moving quickly around the Web searching for information and making hasty decisions about which pages or bits of pages might be useful.

The aim of BrookesTalk is to support users in this type of activity where speedy decisions about the usefulness of a page or part of a page are paramount.

\section{WHAT IS BROOKESTALK?}

BrookesTalk is a small speech output browser which is independent of conventional browsers and also independent of text to speech software applications. It uses Microsoft speech technology.

It includes the functionality of a standard Web browser for the blind such as pwWebSpeak(ä) in that it can break up the text part of a Web page into headings and links and read out paragraphs etc. However the main aim is to provide an orientation tool for blind users. Most particularly to offer a range of tools that will provide a synopsis of a Web page to help the user decide whether it will be useful to them or not. BrookesTalk offers a virtual toolbar driven by function keys. The functions available provide a quick way of knowing what the page is about. Users can select from a list of headings, list of links, list of keywords, 
list of bookmarks, list of scratchpad entries, a summary of the page, abridged text, and can also reach and read out chunks of text which are organised hierarchically under headings. It is expected that the user will pick tools from the virtual toolbar which complement one another for the particular page under review. The keyword list contains words found using accepted information retrieval techniques [1]. Abridged text is compiled from sentences found using key trigrams.

The scratchpad allows users to save any sentence they are listening to which, simply by pressing a key. They can then playback lists of sentences linked to particular pages.

The summary of the page includes author defined keywords, the number of words in a page, the number of headings and the number of links.

\subsection{Keywords}

The following examples of keyword extractions were found for three different Web pages.

\begin{tabular}{|c|c|c|c|}
\hline Document title & Total no.of words & Keywords & Document subject \\
\hline $\begin{array}{l}\text { Thinking of getting a } \\
\text { border collie? }\end{array}$ & 1174 & $\begin{array}{l}\text { border } \\
\text { collie } \\
\text { people } \\
\text { collies } \\
\text { dog } \\
\text { exercise } \\
\text { dogs }\end{array}$ & $\begin{array}{l}\text { Information for } \\
\text { prospective border collie } \\
\text { owners }\end{array}$ \\
\hline $\begin{array}{l}\text { Comet Hale-Bopp Home } \\
\text { Page (JPL) }\end{array}$ & 772 & $\begin{array}{l}\text { comet } \\
\text { hale } \\
\text { image } \\
\text { January } \\
\text { images } \\
\text { vodniza }\end{array}$ & $\begin{array}{l}\text { Information about comet } \\
\text { Hale-Bopp }\end{array}$ \\
\hline $\begin{array}{l}\text { Dennis' Information } \\
\text { Retrieval Page }\end{array}$ & 279 & $\begin{array}{l}\text { information } \\
\text { indexing } \\
\text { IR } \\
\text { pages } \\
\text { index } \\
\text { search } \\
\text { engines }\end{array}$ & $\begin{array}{l}\text { General information } \\
\text { about information } \\
\text { retrieval and links to } \\
\text { related sites }\end{array}$ \\
\hline
\end{tabular}

Table 1 Examples of Keyword Extractions

\subsection{Finding Key Trigrams}

When keywords are used the contextual information imparted by the position of a word in a sentence is lost. Extraction of three word key phrases or trigrams preserves word position information.

The trigram module added to BrookesTalk takes the printable text of the document and from it constructs the set of word level trigrams. To reduce the number of word-level mismatches due to the normal changes in spelling required by grammar, each element of a trigram was assigned the stem of a word rather than the word itself. The trigrams presented were ranked by frequency.

High frequency trigrams occur twice, low frequency trigrams once with little to distinguish between them. Many of the words in the trigrams are noise words which are required for grammatical correctness and are not content bearing.

A summation of frequency of trigram, number of content words in the trigram and number of keywords in the trigram appears as the score for the trigram as in Table 2. which shows key trigrams for the RNIB Web page 'Equal Opportunities Policy' 


\begin{tabular}{|l|l|l|l|l|l|}
\hline Trigram & Frequency & Content - words & Keywords & Score \\
\hline $\begin{array}{l}\text { equal } \\
\text { opportu } \\
\text { n polici }\end{array}$ & 2 & 3 & 3 & 8 & \\
\hline $\begin{array}{l}\text { equal } \\
\text { opportu } \\
\text { n and }\end{array}$ & 2 & 2 & 2 & 6 & \\
\hline $\begin{array}{l}\text { servic } \\
\text { deliveri } \\
\text { and }\end{array}$ & 2 & & 2 & & \\
\hline $\begin{array}{l}\text { it is } \\
\text { commit }\end{array}$ & 2 & 2 & 0 & 3 \\
\hline $\begin{array}{l}\text { is } \\
\text { commit } \\
\text { to }\end{array}$ & 2 & 1 & 0 & 3 \\
\hline
\end{tabular}

Table 2

\subsection{Abridged Text}

Abridged text was created by computing the highest scoring key trigrams of a page, and using the sentences in which the trigrams appeared to provide a summary. Summaries on average worked out to be $20 \%$ of the size of the original text.

\section{EVALUATION}

Preliminary experiments were performed to assess the usefulness of the keyword list as an indicator of page content compared to the headings list or the links list.

\subsection{Keyword Evaluation}

The strength of adding keywords to the BrookesTalk menubar is that it provides more flexibility for the user in summarising the Web page. If the author has truly encapsulated the meaning of subsections of the page in headings then headings should provide a significantly better indicator of page content than keywords. Often however headings are represented as images which do not provide speech output, or are eye-catching rather than informative. In these cases keywords might provide a better summary.

The effectiveness of the extracted keywords in summarising the content of a document was evaluated in terms of recall, the proportion of relevant keywords extracted from the document, and precision, the proportion of extracted keywords that are actually relevant. It is assumed that the more effective the system the more it will satisfy the user. It is also assumed that precision and recall are sufficient for the measurement of effectiveness [2].

User's perception of the usefulness of the representation was also measured by asking them to evaluate the usefulness of describing a Web page using the three different types of summary representations, headings, links/anchors, and keywords.

It is acknowledged that there are differences in the way blind and sighted users listen to and interpret synthetic speech. The issue was not addressed in this preliminary work on keywords. Sighted subjects were also used in this preliminary evaluation because of the possibility of using BrookesTalk over the telephone for Web access [3].

Twenty subject users were shown the different representations for six different Web pages. The pages were chosen to maximise variability. Subjects gave a score between 0 and 5 for each representation. The sum of the scores for each representation, together with the percentage of the total score it 
represented, was taken to give an indication of its effectiveness, results are shown in Table 3.

It can be seen that users perceived that keywords provide a considerable improvement on the use of links to orientate users to Web pages. Headings gave the best score but the score for keywords was not significantly different and increased confidence in our methods.

\begin{tabular}{lcl}
\hline & Total Score & $\begin{array}{c}\text { Percentage of } \\
\text { available marks }\end{array}$ \\
\hline Headings & 406 & 58.09 \\
Anchors & 313 & 45.24 \\
Keywords & 392 & 55.28 \\
\hline
\end{tabular}

Table 3. Scores for different representations

\subsection{Prototype BrookesTalk Evaluation}

The prototype BrookesTalk was used by workers at the Royal National Institute for the Blind (RNIB).

\subsubsection{The BrookesTalk environment}

BrookesTalk uses different voices for conceptually different parts of a Web page. This was appreciated by most but described as irritating by another. We plan to make different voices optional in the future. Users felt it would be useful to have the visual equivalent of the spoken page available at the same time so that sighted co-workers could be called in for clarification or work cooperatively with the blind worker.

\subsubsection{Functionality of BrookesTalk}

Users were observed to rely heavily on one function rather than move between different summarising representations. They had been encouraged to try using the different 'views' of the page to complement one another. The comment was made that you usually know what type of page you are searching for, research work, entertainment, product details etc and therefore know how useful headings are going to be. Surprisingly one user orientated himself by using the movement between links key $90 \%$ of the time. We had not anticipated that he would build his conceptual model of the page by looking at what was behind it. This approach will be investigated fully!

The summary function received most criticisms. It seldom reflected the contents of the whole page. Trigram analysis easily picks out the wrong trigrams as being significant. Important headings were frequently omitted from the summary. It was suggested that trigrams should be weighted if they appear in the title or headings.

\subsubsection{The global picture}

Users at RNIB saw the value of using a tool such as BrookesTalk to sort through what they referred to as the 'increasing pile of paper that arrived on their desks' during the working day. Methods used to translate HTML formatting to speech could easily be applied to other formatted documents. Useful aspects included vocal notification of HTML markup (i.e. if text is a heading, alt-text etc.) and being able to move between headings and start speech from specified points in the text. By incorporating usability at such an early stage in the development process the system is more likely to meet user needs.

\section{CONCLUSIONS}


Preliminary informal unstructured user trials with BrookesTalk. have indicated areas for improvement and provided valuable feedback on the usability of the virtual menubar of functions.

The changes suggested will be incorporated and a larger and more structured evaluation will take place as a collaboration between Oxford Brookes University and RNIB.

\section{REFERENCES}

[1] Luhn, H. P. The automatic creation of literature abstracts. IBM Journal of Research and Development, $2,(1958)$ 159-165.

[2] van Rijsbergen C. J. Information Retrieval, Butterworths, 2 (London 1979).

[3] Zajicek M. and Powell, C. Accessing the World Wide Web by telephone in Proceedings of Designing for our future selves: Human Factors in Telecommunications '97 (Oslo 197), 177-184.

[4] Zajicek, M. and Powell, C. Building a conceptual model of the World Wide Web for visually impaired users in Proceedings of Contemporary Ergonomics (1997) Taylor \& Francis, 270-275

$\underline{\text { Back to publication list. }}$ 